\title{
Sensor Placement for Grid Coverage under Imprecise Detections
}

\author{
Santpal S. Dhillon and Krishnendu Chakrabarty \\ Dept. Electrical \& Computer Engineering \\ Duke University \\ Durham, NC 27708 \\ E-mail: \{ssd5,krish\}@ee.duke.edu
}

\author{
S. S. Iyengar \\ Department of Computer Science \\ Louisiana State University \\ Baton Rouge, LA 70803 \\ E-mail: iyengar@bit.csc.lsu.edu
}

\begin{abstract}
We present a resource-bounded optimization framework for sensor resource management under the constraints of sufficient grid coverage of the sensor field. We offer a unique "minimalistic" view of distributed sensor networks in which sensors transmit/report a minimum amount of sensed data. The proposed theory is aimed at optimizing the number of sensors and determine their placement to support such minimalistic sensor networks. We represent the sensor field as a grid (two- or three-dimensional) of points. The optimization framework is inherently probabilistic due to the uncertainty associated with sensor detections. The proposed algorithm addresses coverage optimization under constraints of imprecise detections and terrain properties. The issue of preferential coverage of grid points (based on relative measures of security and tactical importance) is also modeled. Experimental results for an example sensor field with obstacles demonstrate the application of our approach.
\end{abstract}

Keywords: Detection probability, preferential coverage, obstacles, sensor field coverage, terrain modeling.

\section{Introduction}

Sensor placement directly influences resource management and the type of back-end processing and exploitation that must be carried out with sensed data in distributed sensor networks. A key challenge in sensor resource management is to determine a sensor field architecture that optimizes cost, provides high sensor coverage, resilience to sensor failures, and appropriate computation/communication trade-offs. Intelligent sensor placement facilitates the unified design and operation of sensor/exploitation systems, and decreases the need for excessive network communication for surveillance, target location and tracking. Sensor placement therefore forms the essential "glue" between front-end sensing and backend exploitation.
In this work, we present a resource-bounded optimization framework for sensor resource management under the constraints of sufficient grid coverage of the sensor field. The proposed research offers a unique "minimalistic" view of distributed sensor networks in which a minimum number of sensors are deployed, and they transmit/report a minimum amount of sensed data. Intelligent sensor placement ensures that the ensemble of this data contains sufficient information for the data processing center to subsequently query a small number of sensors for detailed information, e.g. imagery and time series data. The proposed theory is aimed at optimizing the number of sensors and their placement to support such minimalistic sensor networks.

In a typical scenario, surveillance authorities have several different types of sensors available, which can be appropriately placed in the sensor field. These sensors differ from each other in their modalities, monitoring range, detection capabilities, and cost. Intelligent sensor deployment strategies are necessary to minimize cost and yet sufficient sensor coverage. In addition, sensor deployment must take into account the nature of the terrain (obstacles such as buildings and trees in the line of vision for IR sensors, uneven surfaces and elevations for hilly terrains, etc.), redundancy due to the likelihood of sensor failures, and the power needed to transmit between deployed sensors and between a deployed sensor and the cluster head.

We represent the sensor field as a grid (two- or three-dimensional) of points. A target in the sensor field is therefore a logical object, which is represented by a set of sensors that see it. An irregular sensor field is modeled as a collection of grids. The optimization framework is however inherently probabilistic due to the uncertainty associated with sensor detections. The proposed algorithms for sensor placement address issues such as coverage optimization under constraints of 
imprecise detections and terrain properties. The issue of preferential coverage of grid points (based on relative measures of security and tactical importance) is also modeled. We limit our discussion in this paper to fixed sensors. Experimental results for an example sensor field with obstacles demonstrate the application of our approach.

Previous research in distributed sensor networking has largely ignored sensor placement issues. Most prior work has concentrated exclusively on efficient sensor communication $[1,2]$ and sensor fusion [3, 4] for a given sensor field architecture. However, as sensors are used in greater numbers for field operation, efficient deployment strategies become increasingly important. Related work on terrain model acquisition for motion planning has focused on the movement of a robot in an unexplored "sensor field" [5]. While knowledge of the terrain is vital for surveillance, it does not directly solve the sensor placement problem.

Self-deployment for mobile sensors based on the notion of potential fields has been presented in [13]. The fields are constructed such that each sensor is repelled by both obstacles and by other sensors, thereby forcing the network to spread itself through the environment. The potential field approach has been shown to achieve good coverage without global maps, communication and explicit reasoning. Area coverage becomes an emergent, system-level property. However self-deployment does not provide a solution for case of static sensors, which need to be deployed in a specific pattern for example for environmental monitoring.

A related problem to deployment in wireless sensor networks is spatial localization [14]. In wireless sensor networks, nodes need to be able to locate themselves in various environments and on different distance scales. This problem, referred to as localization, is particularly important where sensors have not been deployed according to a set pattern e.g. when sensors are thrown from airplanes in a battlefield, for underwater sensors which might move due to drift. A number of techniques for both fine and coarse-grained localization have been proposed recently $[11,14,15]$.

The problem of coverage provided by a given placement of sensors has also been discussed in literature [12]. The problems of coverage and deployment are fundamentally inter-related. In order to achieve deterministic coverage, a static network must be deployed according to a predefined shape.
Thus an optimal deployment of sensors will also provide good area coverage.

Sensor placement on two- and three-dimensional grids was formulated as a combinatorial optimization problem, and solved using integer linear programming $[6,7]$. This approach suffers from two main drawbacks. First, computational complexity makes the approach infeasible for large problem instances. Second, the grid coverage approach relies on "perfect" sensor detection, i.e. a sensor is expected to yield a binary yes/no detection outcome in every case. It is well known however that there is inherent uncertainty associated with sensor readings, hence sensor detections must be modeled probabilistically [3].

There exists a close resemblance between the sensor placement problem and the art gallery problem (AGP) addressed by the art gallery theorem [8]. The AGP problem can be informally stated as that of determining the minimum number of guards required to cover the interior of an art gallery. (The interior of the art gallery is represented by a polygon.) Several variants of AGP have been studied in the literature, including mobile guards, exterior visibility, and polygons with holes. Our sensor placement problem differs from AGP in two fundamental ways: (a) the sensors can have different ranges, unlike in AGP where guards are assumed to have similar capabilities, and (b) unlike the intruder detection by guards, sensor detection outcomes are probabilistic. Other related work includes the placement of a given number of sensors to reduce communication cost [9], optimal sensor placement for a given target distribution [10].

The remainder of the paper is organized as follows. In Section 2, we describe our sensor detection model as well our approach for modeling the terrain. Section 3 describes our procedure for placing sensors to provide adequate coverage of the sensor field. We also show how the placement algorithm can be augmented to provide differential coverage of grid points (based on relative measures of security and tactical importance). Section 4 presents experimental results for various problem instances. A comparison is presented with random sensor placement to highlight the effectiveness of the proposed approach. Finally, Section 5 concludes the paper and describes directions for future work.

\section{Sensor and Terrain Model}

Sensor placement requires accurate yet computationally feasible sensor detection models. In 
this work, we first assume that the sensor field is made up of grid points. The granularity of the grid (distance between consecutive grid points) is determined by the accuracy with which the sensor placement is desired.

We assume that the probability of detection of a target by a sensor varies exponentially with the distance between the target and the sensor. This model is illustrated in Figure 1. A target at distance $d$ from a sensor is detected by that sensor with probability $\mathrm{e}^{-\alpha d}$. The parameter $\alpha$ can be used to model the quality of the sensor and the rate at which its detection probability diminishes with distance. Clearly, the detection probability is 1 if the target location and the sensor location coincide. For every two grid points $i$ and $j$ in the sensor field, we associate two probability values: (i) $p_{i j}$, which denotes the probability that a target at grid point $j$ is detected by a sensor at grid point $i$; (ii) $p_{j i}$, which denotes the probability that a target at grid point $i$ is detected by a sensor at grid point $j$. In the absence of obstacles, these values are symmetric, i.e. $p_{i j}=p_{j i}$. However, we will show later in this section that these values need not be equal in the presence of obstacles.

Note that the choice of a sensor detection model does not limit the applicability of the placement algorithm in any way. The detection model is simply an input parameter to the placement algorithm. Alternative detection models can therefore be considered without requiring a major redesign of the placement algorithm.

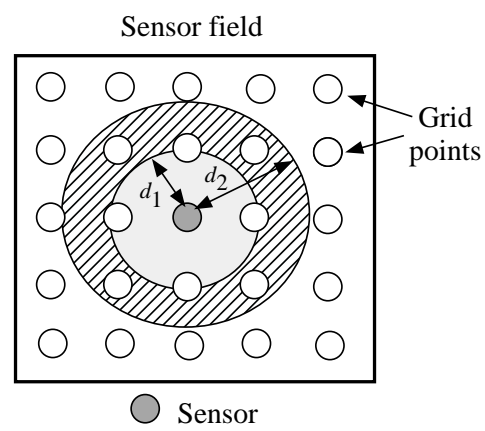

Figure 1. Sensor detection model.

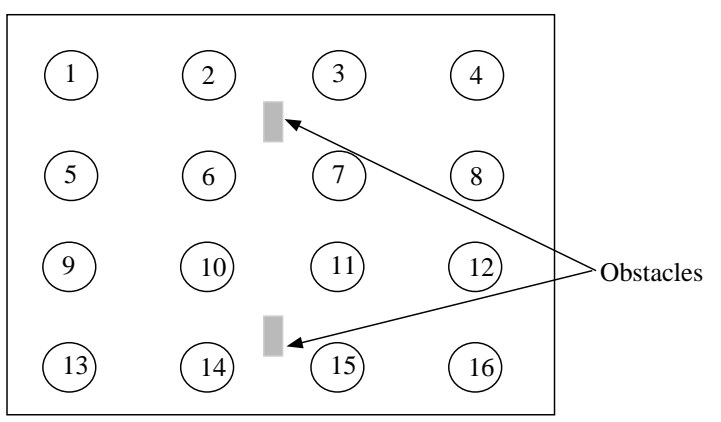

Figure 2: Obstacles in a sensor field.
We next explain how obstacles in the terrain are modeled in this framework. A number of sensors, e.g. IR cameras, require a target to lie in their line of sight. Obstacles cause occlusion and render such sensors ineffective for detection. We assume that knowledge of the terrain is acquired prior to sensor placement, e.g. through satellite imagery. The obstacles are then modeled by altering the detection probabilities for appropriate pairs of grid points. For example, if an object such as a building or foliage is present in the line of sight from grid point $i$ to grid point $j$, we set $p_{i j}=0$. Partial occlusion can also be modeled by setting a non-zero, but small value for the detection probability. The only assumption we are making here is that the obstacles are static, since we do not consider sensor placement or grid coverage as a function of time.

As an example, consider the two obstacles shown in the sensor field of Figure 2. The grid points in this figure are numbered from 1 to 16 . If we assume that these obstacles are symmetric, then they cause $p_{36}, p_{63}, p_{27}, p_{72}$, and a number of other detection probabilities to be rendered zero. It is straightforward to determine if for any two grid points $i$ and $j, p_{i j}$ is affected by an obstacle. Each grid point is associated with a pair of $(x, y)$ coordinates in the plane. Similarly, an obstacle also has associated $(x, y)$ coordinates. We determine the equation of the straight line connecting $i$ and $j$. If the coordinate of the obstacle satisfies this equation, the probability $p_{i j}$ is set to zero.

In many practical instances, obstacles in the sensor field are asymmetric, i.e. $p_{i j}=0$ does not imply that $p_{j i}=0$. This can occur for instance in the case of a hilly terrain. A sensor at a lower elevation is unlikely to detect a target at a higher elevation, but a sensor at a higher elevation can detect a target a lower elevation. This scenario can be easily modeled in our framework by using appropriate detection probability values.

\section{Sensor Placement Algorithm}

In this section, we describe our algorithm for sensor placement for a given set of detection probabilities in a sensor field (both with and without obstacles). The goal of the sensor placement algorithm is to determine the minimum number of sensors and their locations such that every grid point is covered with a minimum confidence level. We use the term coverage threshold to refer to this confidence level. The coverage threshold $T$ is provided as an input to the placement algorithm. Our objective is to ensure 
that every grid point is covered with probability at least $T$.

We begin by generating a sensor detection matrix $D=\left[p_{i j}\right]$ for all pairs of grid points in the sensor field. For an $n$ by $n$ grid, we have a total of $n^{2}$ grid points, hence the matrix $D$ consists of $n^{2}$ rows and $n^{2}$ columns, and a total of $n^{4}$ elements.

From the sensor detection matrix $D$, we determine the miss probability matrix $M=m_{i j}$, where $m_{i j}=1-p_{i j}$. We do not directly use $D$ in our sensor placement algorithm. Instead, we use the entries in the miss probability matrix $M$. The sensor placement algorithm uses a greedy heuristic to determine the best placement of one sensor one at a time. The algorithm is iterative, and it places one sensor in the sensor field during each iteration. It terminates either when a preset upper limit on the number of sensors is reached, or sufficient coverage of the grid points is achieved.

We define the vector $M^{*}=\left(M_{1}, M_{2}, \ldots, M_{N}\right)$ to be the set of miss probabilities for the $N=n^{2}$ grid points in the sensor field. An entry $M_{i}$ in this vector denotes the probability that grid point $i$ is not collectively covered by the set of sensors placed in the sensor field. At the start of the placement algorithm, the vector $M$ is initialized to the all-1 vector, i.e. $M^{*}=(1$, $1, \ldots, 1)$. Each sensor placed in the sensor field decreases one or more entries in this vector. Placement of a sensor also decreases the size of miss probability matrix by one as the corresponding row and column in the miss probability matrix become redundant. The pseudocode steps of the sensor placement algorithm are outlined below. Let $M_{\min }=$ $1-T$ be the maximum value of the miss probability that is permitted for any grid point.

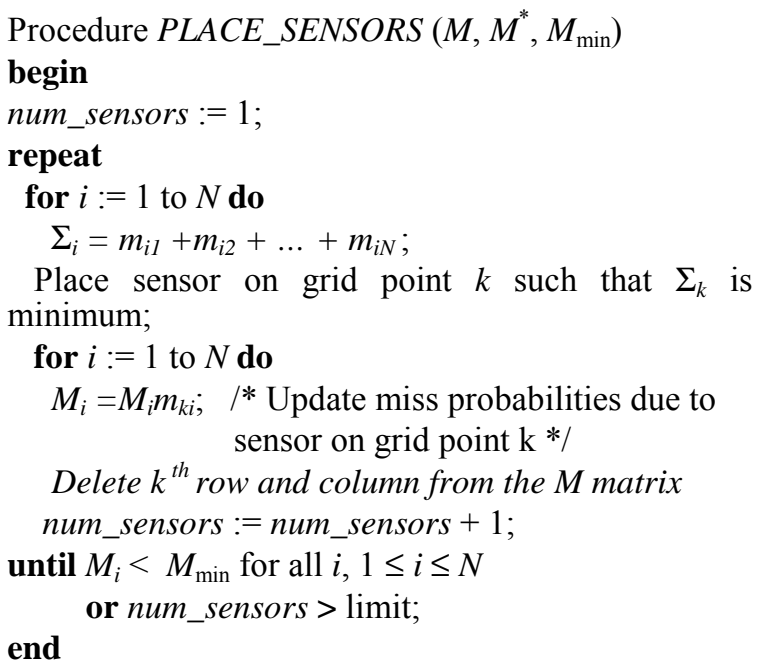

The above pseudocode description makes the implicit assumption that sensor detections are independent, i.e. if a sensor detects a target at a grid point with probability $p_{1}$, and another detects the same target at that grid point with probability $p_{2}$, then the miss probability for the target is $\left(1-p_{1}\right)\left(1-p_{2}\right)$. As part of future work, we will model the realistic scenario when sensor readings are correlated.

Note that the effectiveness of grid coverage due to an additional sensor is measured in the PLACE_SENSORS procedure by the $\Sigma_{i}$ parameter. This approach attempts to evaluate the global impact of an additional sensor by summing up the changes in the miss probabilities for the individual grid points. Alternative approaches such as focusing to minimize the weakest link $\left(\max \left\{\Sigma_{i}\right\}\right)$ can also be considered; however, we did not evaluate such approaches in this work.

We next show how the proposed approach for sensor placement facilitates preferential coverage of grid points. In a typical military force protection or civilian defense scenario, certain installations need to be given additional protection. Such installations might include nuclear power plants, command headquarters, or civilian administration centers.

In order to model preferential coverage, we assign a different protection probability $p r_{i}$ to each grid point $i$. The miss probability threshold for grid point $i$ is then expressed as $M_{\min }^{i}=1-p r_{i}$. The procedure PLACE_SENSORS is modified such that the termination criterion of the repeat/until loop is based on checking that the individual miss probability threshold of each grid point has been reached.

\section{Experimental Results}

In this section, we present results on a number of case studies on sensor placement. In order to evaluate the effectiveness of the PLACE_SENSORS procedure, we used random placement of sensors as a baseline. Our first observation is that if there are no obstacles in the sensor field and all sensors are considered identical, random placement is as effective as PLACE_SENSORS. This is hardly unexpected since the regularity of these problem instances render them especially amenable for random placement. We next show that random placement performs significantly worse when the sensor field contains obstacles and when preferential coverage is desired. 


\section{Case Study 1}

Our first case study was based on a 2dimensional grid with 20 grid points in each dimension for a total of 400 grid points. We used $\alpha=$ 0.5 in calculating the detection probability values for this example. A number of random obstacles were incorporated into the model, as a result of which a significant number of detection probabilities were either made zero or considerably reduced compared to the values obtained from our detection model.

The results plotted in Figure 3 show that far fewer sensors are required when the PLACE_SENSORS procedure is used. The savings over random placement are especially significant when the miss probability threshold $M_{\min }$ is low, i.e. more effective coverage of the sensor field is desired.

\section{Case Study 2}

Our second case study was for a 2-dimensional grid with 8 grid points in each dimension for a total of 64 grid points. We used $\alpha=0.6$ in calculating the detection probability values for this example. Two obstacles were deterministically placed in the sensor field at specific locations for this example.

The results plotted in Figure 4 show that fewer sensors are required when the PLACE_SENSORS procedure is used when the miss probability threshold $M_{\min }$ is low, i.e. more effective coverage of the sensor field is desired. For larger values of the threshold, random placement seems to perform

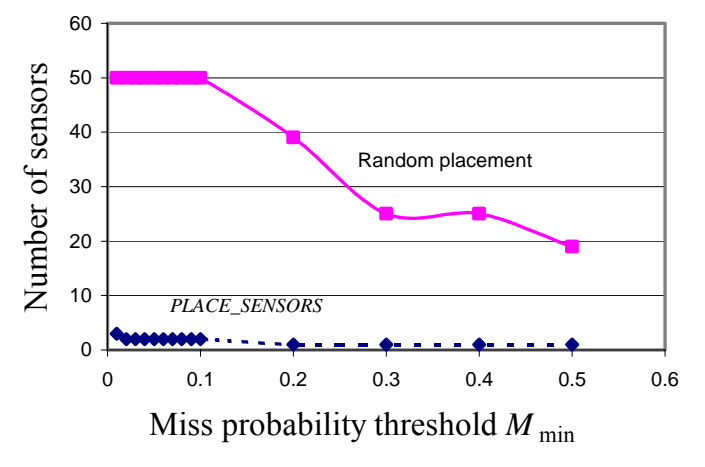

Figure 3: Results for case study 1 ( 20 by 20 grid with randomly-placed obstacles).

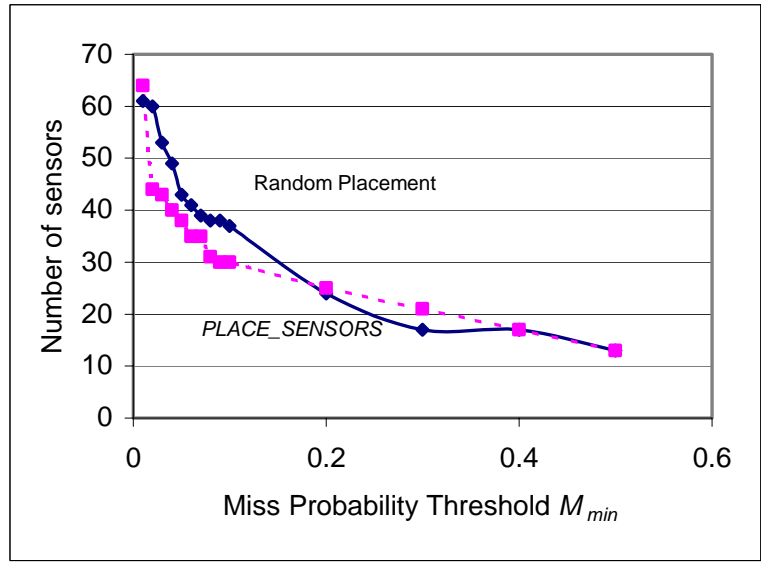

Figure 4: Results for case study 2 ( 8 by 8 grid with two obstacles).

better; nevertheless, we expect sensor placement and grid coverage to be of less importance when the threshold $M_{\min }$ is high.

\section{Case Study 3}

In our third case study, we again used a 2dimensional grid with 8 grid points for a total of 64 grid points. Once again, we used $\alpha=0.6$ in calculating the detection probability values. In this case however, we placed three obstacles in the sensor field at specific locations with the detection probability set to zero when any obstacle lies on the straight line between the two sensors. The results are shown in Figure 5. PLACE_SENSORS outperformed random placement for smaller values of $M_{\min }$, and was no worse for larger values of $M_{\min }$ primarily due to the additional obstacle in the sensor field.

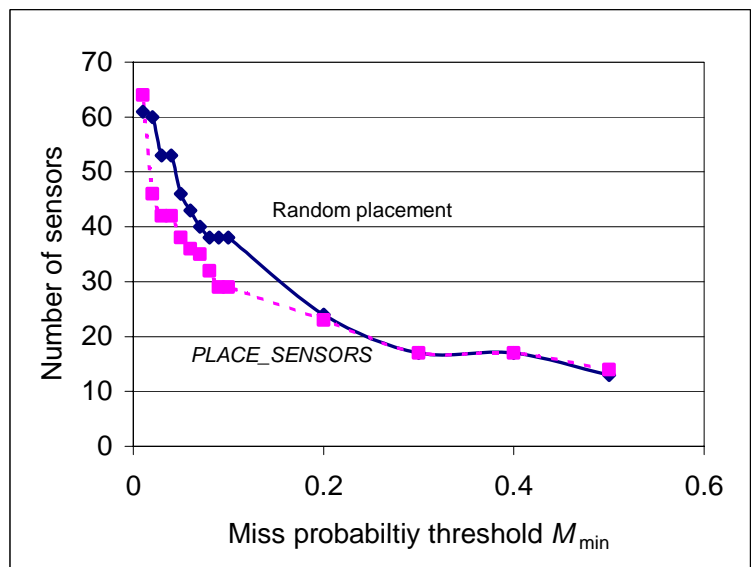

Figure 5: Results for case study 3 ( 8 by 8 grid with three obstacles). 


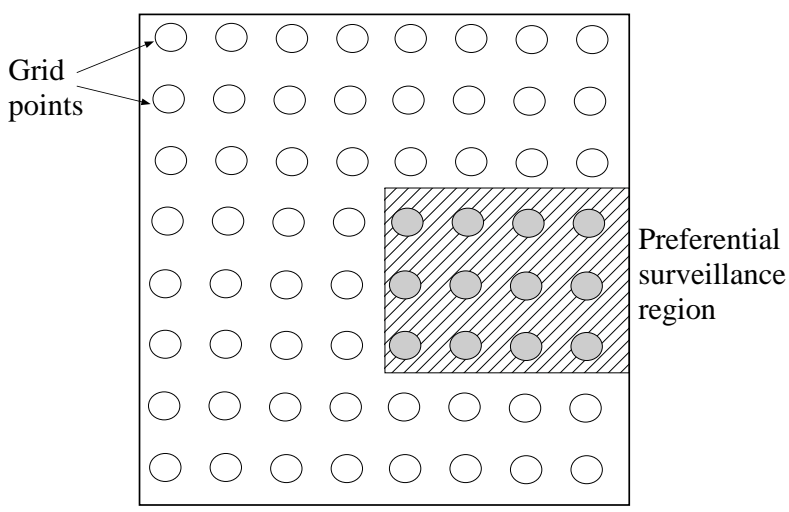

Figure 6: Preferential coverage in sensor field for case study 4.

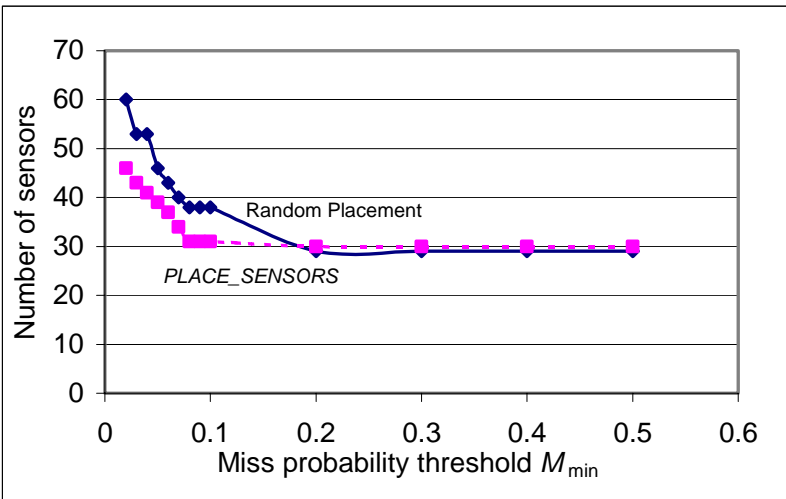

Figure 7:Results for case study 4 ( 8 by 8 grid with four obstacles and preferential coverage).

\section{Case Study 4}

In our fourth case study, we considered an 8 by 8 grid with four preset obstacles. We used $\alpha=0.6$ in calculating the detection probability values. In addition, we considered preferential coverage in this case study. A subset of grid points (marked in Figure 6) was required to be covered with a low miss probability threshold $M_{\min }=0.01$. The threshold for the other grid points in the sensor field was varied to determine the number of sensors needed for random placement and by the PLACE_SENSORS procedure. The results in Figure 7 show that PLACE_SENSORS significantly outperforms random placement for smaller values of the (variable) miss probability.

\section{Case Study 5}

This case study was for a 10 by 10 grid with $\alpha=0.5$. We do not place any obstacles in this grid. Figure 8 shows that PLACE_SENSORS better than random placement for all values of $M_{\min }$ that we considered.

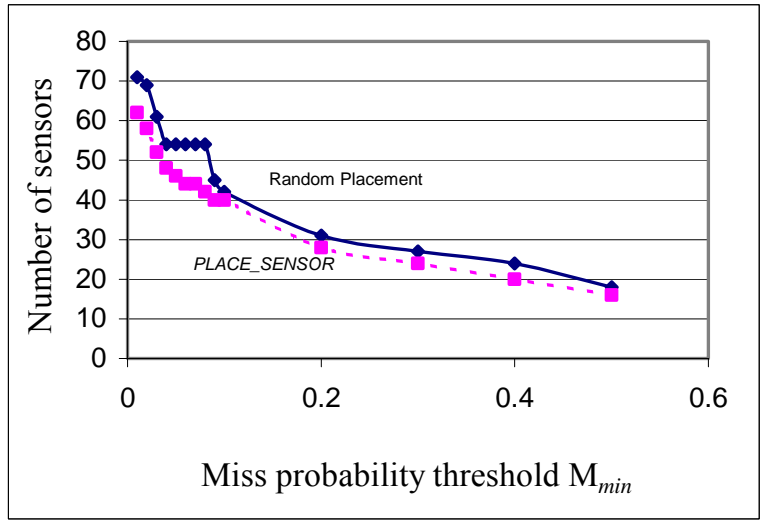

Figure 8: Results for case study 5 (10 by 10 grid with no obstacles).

Case Study 6: Our last case study is for a 10 by 10 grid with $\alpha=0.5$. We placed four obstacles in this grid with the detection probability set to zero when any obstacle lies on the straight line between the two sensors. The results in Figure 9 show that PLACE_SENSORS performs better than random placement even for large values of $M_{\min }$ in this example.

\section{Conclusions}

We have formulated an optimization problem on sensor placement, wherein a minimum number of sensors are deployed to provide sufficient grid coverage of the sensor field. This approach offers a unique "minimalistic" view of distributed sensor networks in which a minimum number of sensors are deployed and sensors transmit/report a minimum amount of sensed data.

We have presented a polynomial-time algorithm to optimizing the number of sensors and determine their placement to support such minimalistic sensor networks.

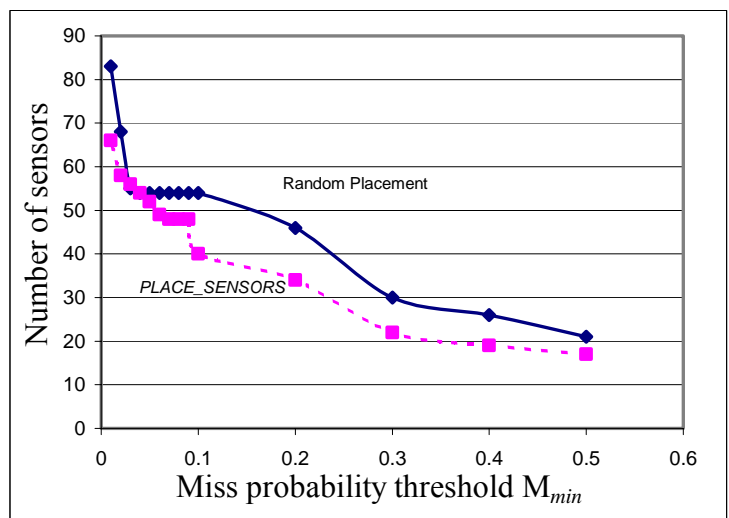

Figure 9: Results for case study 5 (10 by 10 grid with four obstacles). 
We have represented the sensor field as a grid (twoor three-dimensional). The optimization framework is inherently probabilistic due to the uncertainty associated with sensor detections. The proposed algorithm addresses coverage optimization under constraints of imprecise detections and terrain properties. The issue of preferential coverage of grid points (based on relative measures of security and tactical importance) has also been modeled. Several case studies for example sensor fields with obstacles and preferential coverage that the proposed approach significantly outperforms random placement of sensors.

We are currently extending this work to the case of correlated sensor detections and more realistic sensors where the observable domain is defined by minimum and maximum range and an angular width. Also, we would like to address the issues of moving sensors and obstacles in near future. We are also exploring alternative strategies for selecting a sensor in each step of the PLACE_SENSORS procedure. Finally, we are examining how in addition to determining a sensor location in each iteration, PLACE_SENSORS can also determine an appropriate sensor from a set of candidate sensors of the same modality. This work is expected to pave the way for an integrated framework for sensor placement that incorporates power management and fault tolerance.

\section{Acknowledgements}

This research was supported in part by DARPA under grant number N66001-00-1-8946, and in part by Office of Naval Research under grant number N00014-01-1-0712.

\section{References}

[1] J. M. Kahn, R. H. Katz and K. S. J. Pister, "Mobile networking for smart dust", ACM/IEEE International Conference on Mobile Computing and Networks, pp. 271-278, 1999.

[2] D. Estrin, R. Govindan, J. Heidemann and S. Kumar, "Next century challenges: Scalable coordination in sensor networks", Proc. ACM/IEEE International Conference on Mobile Computing and Networks, 1999.

[3] R. R. Brooks and S. S. Iyengar, Multi-Sensor Fusion: Fundamentals and Applications with Software, Prentice Hall PTR, Upper Saddle River, NJ, 1998.

[4] S. S. Iyengar, L. Prasad and H. Min, Advances in Distributed Sensor Technology, Prentice-Hall, Englewood Cliffs, NJ, 1995.
[5] N. S. V. Rao, S. S. Iyengar, B. J. Oomen and R. L. Kashyap, On terrain model acquisition by a point robot amidst polyhedral obstacles, IEEE Journal of Robotics and Automation, vol. 4, pp. 450-455, August 1988.

[6] K. Chakrabarty, S. S. Iyengar, H. Qi and E. Cho, "Grid coverage for surveillance and target location in distributed sensor networks", IEEE Transactions on Computers, vol. 51, 2002 (to appear).

[7] K. Chakrabarty, S. S. Iyengar, H. Qi and E. Cho, "Coding theory framework for target location in distributed sensor networks", Proc. International Symposium on Information Technology: Coding and Computing, pp. 130-134, 2001.

[8] J. O'Rourke, Art Gallery Theorems and Algorithms, Oxford University Press, New York, NY, 1987.

[9] T. Kasetkasem and P. K. Varshney, "Communication Structure Planning for Multisensor Detection Systems", IEE Proc. Radar, Sonar and Navigation, vol. 148, pp. 2-8, Feb. 2001.

[10] D. E. Penny, "The automatic management of multi-sensor systems", Proc. International Conference on Information Fusion (FUSION 1998), July 1998.

[11] N. Bulusu, J. Heidemann and D. Estrin, "Adaptive beacon placement", Proc. Int. Conf. Distributed Computing Systems, pp. 489-498, 2001.

[12] S. Meguerdichian, F. Koushanfar, M. Potkonjak and M. B. Srivastava, "Coverage problems in wireless ad-hoc sensor networks", Proc. IEEE Infocom, vol 3, pp. 1380-1387, 2001.

[13] A. Howard, M. J. Matarić, and G. S. Sukhatme, "Mobile sensor network deployment using potential fields: A distributed, scalable solution to the area coverage problem", Proc. International Symposium on Distributed Autonomous Robotics Systems, June 2002, (to appear).

[14] N. Bulusu, J. Heidemann and D. Estrin, "GPSless low-cost outdoor localization for very small devices", IEEE Personal Communication Magazine, vol. 7, no. 5, pp. 28-34, Oct 2000.

[15] J. Heidemann and N. Bulusu, "Using geospatial information in sensor networks", Proc. CSTB workshop on Intersection of Geospatial Information and Information Technology, October 2001. 